\title{
Investigating the protective properties of milk phospholipids against ultraviolet light exposure in a skin equivalent model
}

\author{
Ashley Russell ${ }^{\mathrm{a}}$, Andrea Laubscher ${ }^{\mathrm{b}}$, Rafael Jimenez-Flores ${ }^{\mathrm{b}}$, Lily H. Laiho ${ }^{\mathrm{a}}$ \\ ${ }^{a}$ Biomedical Engineering Department, California Polytechnic State University, San Luis Obispo, CA \\ USA 93407 \\ ${ }^{\mathrm{b}}$ Dairy Products Technology Center, California Polytechnic State University, San Luis Obispo, CA \\ USA 93407
}

\begin{abstract}
Current research on bioactive molecules in milk has documented health advantages of bovine milk and its components. Milk Phospholipids, selected for this study, represent molecules with great potential benefit in human health and nutrition. In this study we used confocal reflectance and multiphoton microscopy to monitor changes in skin morphology upon skin exposure to ultraviolet light and evaluate the potential of milk phospholipids in preventing photodamage to skin equivalent models. The results suggest that milk phospholipids act upon skin cells in a protective manner against the effect of ultraviolet (UV) radiation. Similar results were obtained from MTT tissue viability assay and histology.
\end{abstract}

Keywords: skin, multiphoton, confocal, MTT assay, histology, milk phospholipid, engineered tissue

\section{INTRODUCTION}

The American Cancer Society estimates that there were 68,720 new cases of melanoma of the skin in 2009, as well as more than 1 million newly diagnosed cases of basal and squamous cell carcinoma among Americans[1]. The prevention of photoaging is desirable as photoaging can eventually lead to skin cancer. Skin is the largest organ of the body encompassing the most surface area[2]. The body uses it to provide structural support, temperature control, and protection of the inner organs and tissue. Photoaging experiments are often carried out in animal models[3-5]. However, a promising alternative to study in animal models is the use of skin equivalent models. In this study, we used skin equivalent models, which are a non-transformed, human-derived, metabolically active, 3 dimensional organotypic in vitro skin model featuring the epidermis Companies have used this model to analyze their products, including performing skin irritation tests[6]. According to a European study of in vitro alternatives for measuring skin irritation potential, EpiDerm received a coefficient correlation of 0.94[7].

The Ultraviolet (UV) spectrum has been known to cause damage in skin in varying degrees. Although UVA does not induce a sunburn, DNA damage does result deep within the skin causing oxidative stress to the cells thus increasing the risk of malignant melanomas [8, 9]. UVB is essential for production of vitamin D but in excess amounts, it will cause a sunburn[10]. UVB primarily affects the epidermis. In this study, tissue engineered skin was exposed to $60 \mathrm{~mJ} / \mathrm{s}$ of UVB. This dose of UVB is equivalent to triple the minimum erythema dose (MED), the minimal single dose of UVR that is required to produce erythema in exposed skin[11].

Sphingomyelin is a naturally occurring phospholipid found in bovine milk, and it has been shown in mice that a diet rich in bovine phospholipids improves epidermal function[12]. In the study, thirteen-week-old hairless mice were separated into two groups; a control group and an experimental group, which was fed a phospholipid-rich diet for six weeks.

Multiphoton Microscopy in the Biomedical Sciences X, edited by Ammasi Periasamy, Peter T. C. So, Karsten König, Proc. of SPIE Vol. $7569,75692 Z$ - @ 2010 SPIE · CCC code: 1605-7422/10/\$18 · doi: 10.1117/12.845803 
Results showed increased levels of stratum corneum hydration and ceramides as well as decreased transepidermal water loss for the phospholipid-rich diet experimental group[12]. These results support the proposed theory of increased epidermal function due to sphingomyelin and contribute to our hypothesis. Recently, researchers have attributed the highly bioactive molecules sphingolipids and phospholipids to the epidermis' stratum corneum barrier function[13-16], and the regulation of cell growth, differentiation and apoptosis[17, 18].

In this study, we investigate the acute inflammatory response of skin when exposed to ultraviolet radiation. By studying the skin equivalent models, we are developing a method that is sensitive to the on-set of photoaging in order to study photoaging and develop methods to minimize or protect against its effects. This will enable us to study the protective effects of milk phospholipids and begin to understand the mechanisms by which the benefits occur. We use four different methods to study the protective effects: confocal reflectance microscopy, MTT assay, histology, and multiphoton microscopy. Confocal reflectance and multiphoton microscopy allows the observation of functional, living tissue without the destructive effects of other viewing and analysis methods. The MTT Tissue Viability Assay is a colormetric assay system that measures the reduction of 3-(4,5-Dimethylthiazol-2-yl)-2,5-diphenyltetrazolium bromide in order to measure the viable cells. Histology provides information as to the morphology, architecture, and composition of the tissue.

\section{METHODS}

\subsection{Preparation of Tissue}

The skin equivalent model (EpiDerm, Mattek Corporation, Ashland, MA) was incubated at $37^{\circ} \mathrm{C}$ for 1 hour in $0.9 \mathrm{ml}$ of maintenance media. The samples were then transferred into the corresponding media for $24 \mathrm{hrs}$ in $37^{\circ} \mathrm{C}$. See Table 1 for Sample Group Descriptions. After the 24 hour incubation period, the corresponding samples were exposed to ultraviolet light. After the dosing, all samples were transferred into fresh media and incubated until experiments were performed.

\subsection{Preparation of the Milk Phospholipid Solution}

A $1 \%$ milk phospholipid solution was made using MatTek media and milk phospholipid powder (Phospholipid Concentration 700, Finterra Ltd, Edgercumbe). In order to thoroughly mix it, the solution was brought to $55^{\circ} \mathrm{C}$. It was then filtered with $10 \mathrm{ml}$ syringes to sterilize the solution before it was introduced to the tissue samples.

\subsection{Ultraviolet Light Exposure}

The skin tissue was exposed to $60 \mathrm{~mJ} / \mathrm{s}$ of $302 \mathrm{~nm}$ light using a UV lamp (QPanel Industries, UV340). Only the UV and UV + Milk samples were exposed to ultraviolet light - see Table 1 for Sample Group Descriptions.

\begin{tabular}{|cl|}
\hline Group \# & Description \\
\hline 1 & Control: Tissue incubated with MatTek's maintenance media \\
2 & Milk: Tissue incubated with 1\% milk phospholipid solution \\
3 & UV: Tissue incubated with MatTek's maintenance media before exposure to UV \\
4 & UV + Milk: Tissue incubated with 1\% milk phospholipid solution before UV exposure \\
\hline
\end{tabular}

Table 1. Sample Group Descriptions 


\subsection{Confocal Microscopy}

A laser scanning confocal microscope (FluoView IX81, Olympus) with a 40x oil (UPlanSApo, Olympus) objective was employed to image the tissue. The laser power was set to $3.0 \%$ at $408 \mathrm{~nm}, 3.0 \%$ at $488 \mathrm{~nm}, 4.0 \%$ at $515 \mathrm{~nm}$, and $3.0 \%$ at $559 \mathrm{~nm}$. One sample per group (see Table 1) was imaged immediately after UV exposure. Other rounds of one sample per group were imaged at $6 \mathrm{hrs}$ and then at $24 \mathrm{hrs}$ after UV exposure. The same twelve samples used in the confocal imaging were allocated for the multiphoton imaging as well. The tissue was cut out of its cell culture insert. It was then dipped in phosphate buffered saline (PBS) individually to keep it moist and placed with the stratum corneum side of skin facing toward the coverslip. A gasket (Coverwell, Invitrogen) was positioned in between the coverslip and the slide. The images' signal intensity was found by adding up the pixels using ImageJ software.

\subsection{MTT Tissue Viability Assay}

An additional twelve samples were used in the MTT tissue viability assay. After the initial preparation as stated in section 2.1, we incubated the tissue inserts for $3 \mathrm{hrs}$ in MTT assay solution after being rinsed twice with PBS. Tissue samples were then removed from media, blotted with a kimwipe, and submerged in $2.0 \mathrm{ml}$ of extractant in a 24 -well plate. They were stored at room temperature in the dark overnight. The MTT extractant was mixed well before it was transferred to a 96-well plate. Optical Density was determined by a spectrophotometer (SpectraMax, Molecular Devices) and analyzed by SOFTMax Pro.

\subsection{Histology}

Another twelve tissue samples were used for histological imaging. After the tissue preparation and UV dosing took place, the inserts were cut out of their plastic wells and submerged in the fixative Histochioce. Within 48 hours, the tissues were processed, embedded, and stained. An altered H \& E staining protocol was used where exposure to Hematoxylin was reduced to 2 mins and exposure to the Eosin was reduced to $1 \mathrm{~min}$ and $30 \mathrm{sec}$ in order to accurately identify the structures and artifacts of the tissue.

\subsection{Multiphoton Microscopy}

Twelve tissue samples used in the multiphoton microscopy were prepared similarly to those used in the confocal setup. The laser was set to $755 \mathrm{~nm}$ at $15 \%$ power while using the 40x (UPlanSApo, Olympus) objective. Images were analyzed with ImageJ software. The images' signal intensity was found by adding up the pixels using ImageJ software. 


\section{RESULTS}

\subsection{Confocal Imaging}

Morphological changes were noticed in the most superficial layer of the stratum spinosum at approximately 10 - 30 um deep. Morphological changes were not observed in deeper layers. Figure 1 shows representative images of each sample group demonstrating the observed differences. It is apparent that the UV samples, Figure 1(c), appear significantly different from the other categories of images. There is a significant increase in the number of "spots" seen in Figure 1(c) as compared to the other experiment groups. The samples from the Control, Milk, and UV + milk groups, Figures 1(a), 1(b), and 1(d), appear similarly morphologically with an additional increase in background reflectance from the samples treated with the milk phospholipid solution. In addition, the average intensity was calculated for each of the images. The average intensity for Control was 744.5, Milk was 1285.2, UV was 1676.5, and UV + Milk was 1246.1.

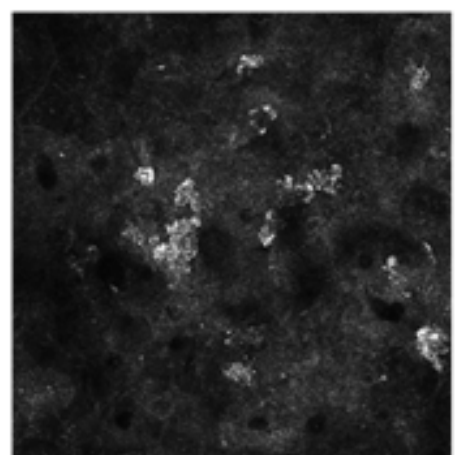

(a)

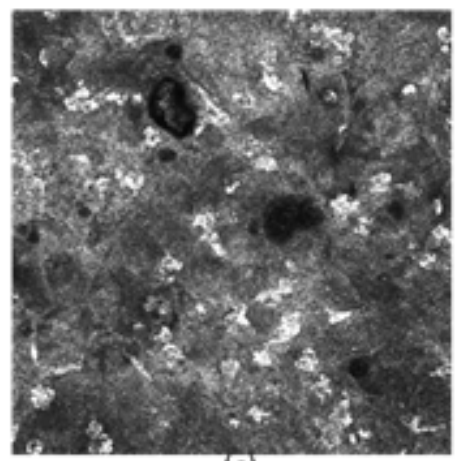

(c)

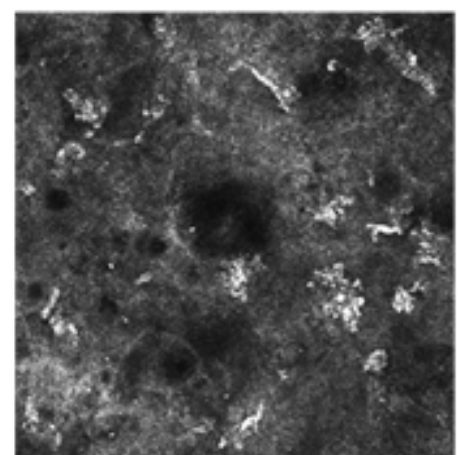

(b)

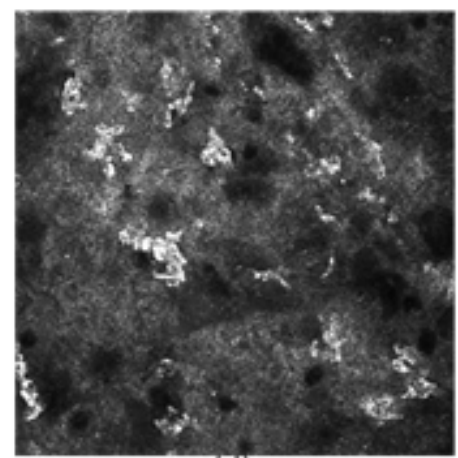

(d)

Figure 1. Confocal reflectance images of the skin equivalent models obtained at approximately 20 um deep within the tissue. These are representative images of (a) Control; (b) Milk; (c) UV; (d) UV + Milk. 


\subsection{MTT Tissue Viability Assay}

The results form the MTT Tissue Viability Assay can be found in Table 2. The results show the measured optical density along with the calculated percent viability. The percent viability is calculated by equation (1) for each corresponding dose.

$$
\text { Percent Viability }=100 *[(\mathrm{OD}(\text { sample }) / \mathrm{OD}(\text { negative control })]
$$

The Control group was used as the negative control for the calculations. The most metabolically active tissue is the UV + Milk group with an optical density (OD) of 1.790. The Milk samples had an optical density of 1.523; the Control group had an optical density of 1.440, and the UV group had an optical density of 1.358 . Two samples had an increase in cell viability: Milk was calculated to be $106 \%$ cell viability and UV + Milk with $124 \%$ cell viability. The UV exposure group resulted with a negative overall viability of $94 \%$.

\begin{tabular}{|lrr|}
\hline Group \# & Optical Density & Percent Viability \\
\hline Control & 1.440 & 100 \\
Milk & 1.523 & 106 \\
UV & 1.358 & 94 \\
UV + Milk & 1.790 & 124 \\
\hline
\end{tabular}

Table 2. Percent Viability using Group 1 (Control) as a negative control. 


\subsection{Histology}

The most superficial part of the tissue is the stratum corneum, which is located on the left-hand side of the histology images. Figure 2(a) is the only sample that still has the cell culture insert upon which it was cultured. Note that sunburned cells are formed in Figure 2(c) (see arrows) as compared to the other images (a), (b), and (d). These same features are not seen in the Control, Milk, or UV + Milk images.

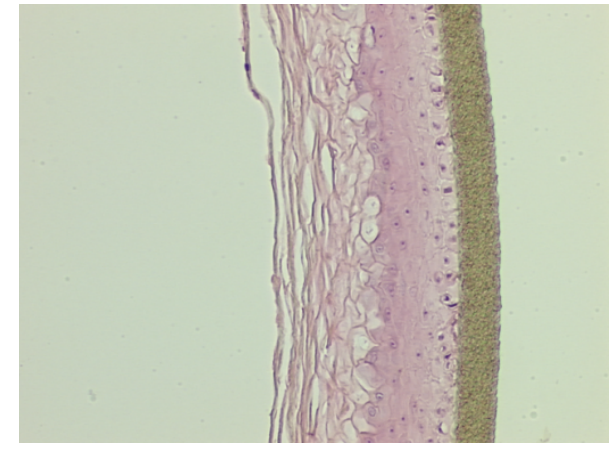

(a)

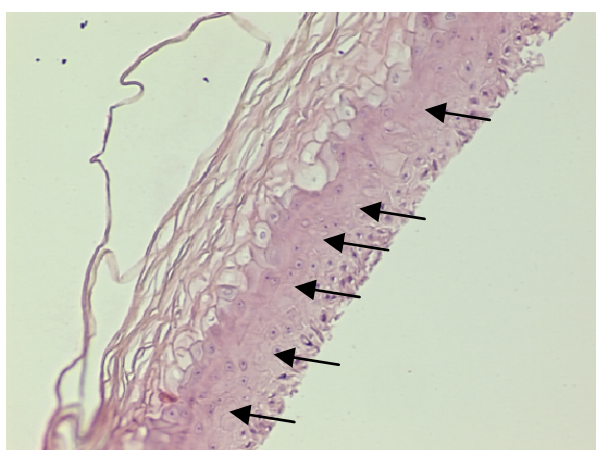

(c)

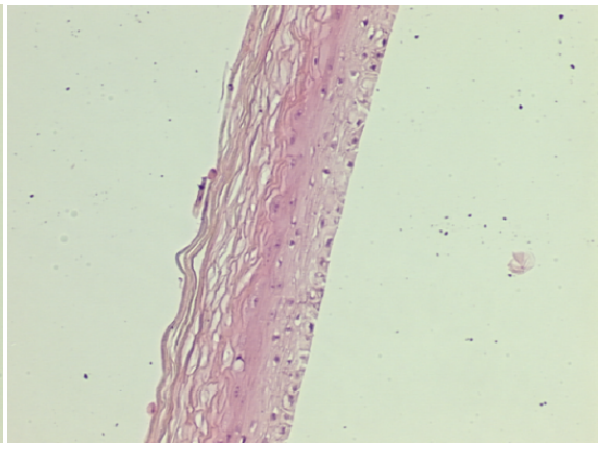

(b)

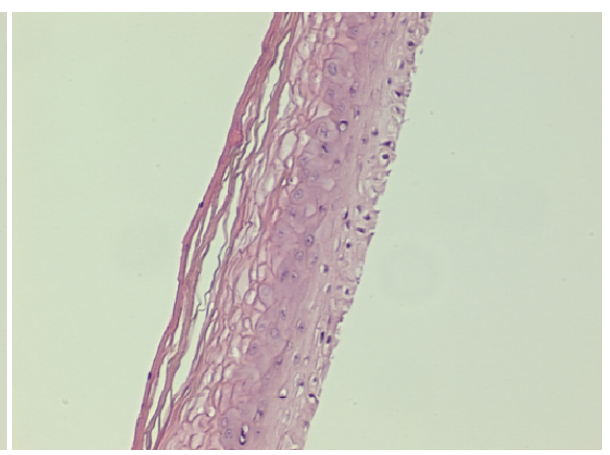

(d)

Figure 2. Representative Hematoxylin and Eosin histology images of (a) Control; (b) Milk; (c) UV; (d) UV + Milk.

\subsection{Multiphoton Imaging}

No significant morphological or biochemical differences were noted in the multiphoton images taken. Representative images from each experiment group are shown in Figures 3(a) - 3(d). 


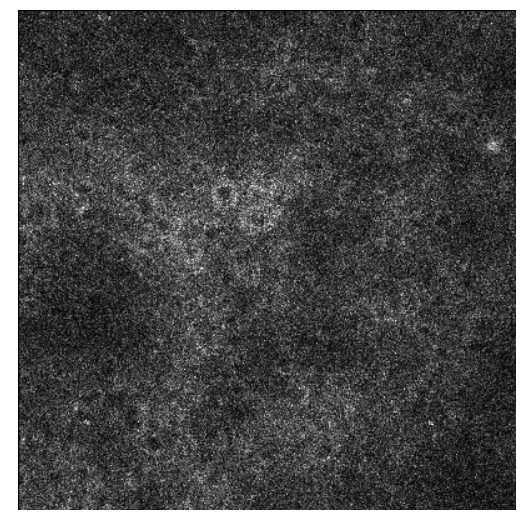

(a)

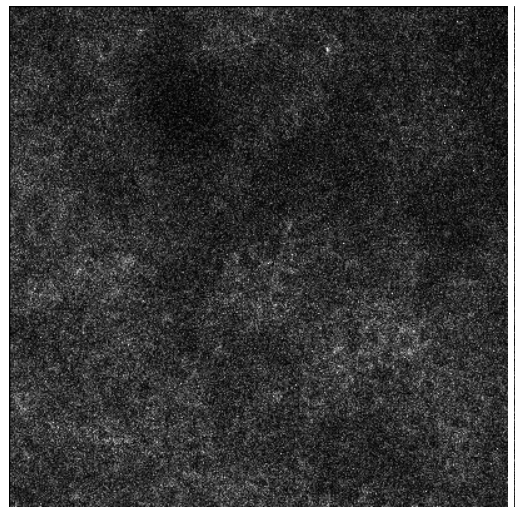

(c)

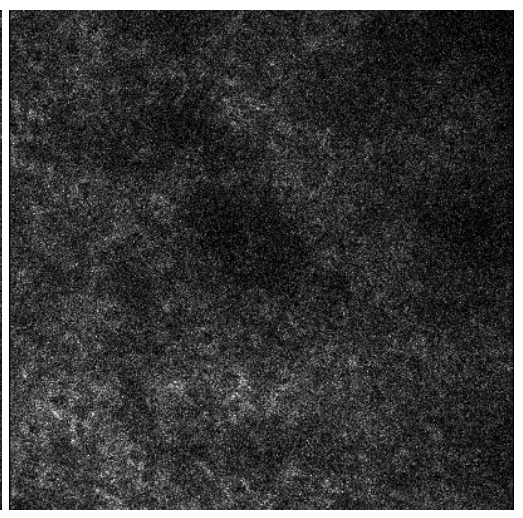

(b)

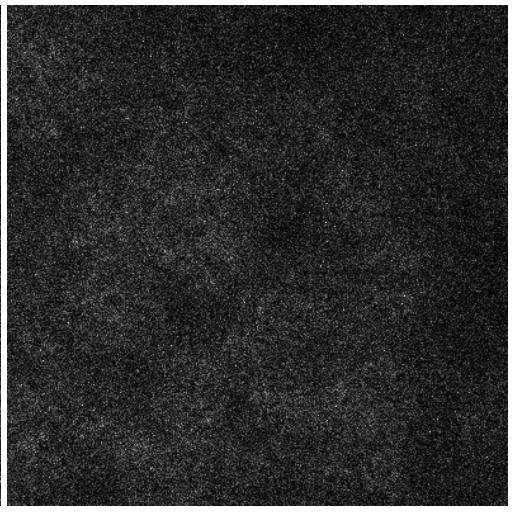

(d)

Figure 3. Representative multiphoton images of the skin equivalent models are equivalent depths for (a) Control; (b) Milk; (c) UV; (d) UV + Milk.

\section{DISCUSSION}

The confocal images shown in Figures 1 (a) - (d) confirm a difference in the tissue morphology between the experiment groups. The UV exposed samples, Figure 1(c), show an increase in reflected light from the sample in the form of additional "spots" as well as background noise. This is also reflected in the increase in average intensity between the UV samples and rest of the experiment groups. As UV exposure at a dosage of $60 \mathrm{~mJ} / \mathrm{s}$ is known to cause damage in skin, we believe the increase in signal and localized spots in the UV images as compared to the Control images indicate the presence of damage. These localized spots could be due to DNA damage or acute damage that results in a localized increase in reflectance. In addition, the Milk and UV + Milk images, Figures 1(b) and 1(d), appear more similar to the Control group, Figure 1(a), than to the UV group, Figure 1(c), indicating little or no damage to the skin equivalent model. As the only difference in media is the presence of the milk phospholipids, a likely explanation is that the milk phospholipid is used by the cell in such a way that assists in the protection and possibly the repair of damage done by the UV. If the milk phospholipid is protecting the skin equivalent, we would expect to see a sample most similar to the control encompassing the least amount of damage just as we do in Figure 1. 
The MTT Tissue Viability Assay results, Table 2, also indicate that the presence of the milk phospholipid increases viability in Milk and UV + Milk samples. As expected, UV exposure is the least viable faction due to UV damage. Samples exposed to the milk phospholipids had higher cell viability than the negative control showing that the milk phospholipid may be helping the cells stay alive and/or protecting the skin. Another reason for the increase in optical density of could be due to the presence of the milk phospholipids themselves in the samples. The milk phospholipids could cause an increase in the optical density being measured.

Histology illustrates that the morphology of the skin indeed does change upon UV exposure and that the existence of the milk phospholipid alone does minimize damage. Figure 2(a) signifies a healthy living tissue including the matrix it grew on. The other histological images do not have the matrix still attached due to its fragility; the other matrices fell off during processing. UV exposure degraded the tissue, causing the stratum corneum to pull away from the rest of the epidermis as seen in Figure 2(c). UV + Milk does not present that issue even though the tissue was exposed to UV. The only difference between UV and UV + Milk is that there are phospholipids present in the later indicating that the milk phospholipids offer protective benefit for the tissue. Another indication signifying that the milk does act in a positive manner is that UV has an increase in the number of nuclei whereas the tissue that was treated with the milk phospholipid remained similar to Control. Both UV exposed groups did exhibit irregularly shaped nuclei; instead of being rounder like Control and Milk samples, the edges appeared to be sharp and jagged, possibly indicating that the DNA was damaged. Since there was significantly less of this damage in the UV + Milk samples as compared to the UV samples, this indicate that the media with milk phospholipids provides some protective properties provided by the addition of milk to the media. Figure 2(c) shows evidence of more globular-like cells in the deeper layers of the epidermis. The other three groups do not display the same characteristics.

The multiphoton microscopy images showed little differences between experiment groups, as shown in Figures 3(a) (d). A previous multiphoton study performed with a homemade multiphoton system did show differences between Control and UV groups. Experiments with milk phopholipids were not performed with the homemade system. This indicates that multiphoton microscopy could be used to image differences in our experiments. However, the current experiment images were obtained with a different system (Olympus FluoView IX81) with internal detectors. The use of internal detectors as opposed to external detectors led to a large loss in signal. Thus reducing the sensitivity of our current system for demonstrating the differences between the various experimental groups. Future work will be performed with the purchase of an external detector system.

Confocal imaging, MTT assay tissue viability, and histology all suggest that the milk phospholipids act in a protective manner against ultraviolet exposure. Current studies are in progress to determine the pathway through which this protective benefit is occurring. Further studies include comet assay to identify the extent of DNA damage and Western blot to comprehend the protein composition of the tissue and whether or not the damage caused by ultraviolet exposure alters protein regulation within the cells. 


\section{REFERENCES}

[1] T. A. C. Society, [Cancer Facts and Figures 2009], Atlanta, GA(2009).

[2] E. Marieb, Hoehn, K., [Human Anatomy and Physiology] Pearson Education, Inc, San Francisco, CA, 153-55 (2007).

[3] S. H. Yasui H, "Age-dependent generation of reactive oxygen species in the skin of live hairless rats exposed to UVA light,” Experimental Dermatology, 12(5), 655-661 (2003).

[4] C. M. Kligman LH, Kligman AM, "An animal model for assessing the effects of chemical peels on photoaged skin," Journal of Dermatological Treatment, 10(1), 37-45 (1999).

[5] U. M. Ichihashi M, Budiyanto A, Bito T, Oka M, Fukunaga M, Tsuru K, Horikawa T, "UV-induced skin damage," Toxicology, 189, 21-39 (2003).

[6] M. Robert, Bissonauth, V., Ross, G., Rauabhia, M., "Harmful effects of UVA on the structure and barrier function of engineered human cutaneous tissue," International Journal of Radiation Biology, 75(3), 317-326 (1998).

[7] [MatTek Corportation].

[8] P. H. Berneburg M, Medve-König K, Pfahlberg A, Gers-Barlag H, Gefeller O, Krutmann J, "Induction of the photoaging-associated mitochondrial common deletion in vivo in normal human skin," The Journal of Investigative Dermatology, 122(5), 1277-83 (2004).

[9] R.-A. A. Budai M, Szabo T, Toth S, Ronto G, Sage E, Grof P, "Effect of UVA radiation on membrane fluidity and radical decay in human fibroblasts as detected by spin labeled stearic acids," Journal of Photochemistry and Photobiology, 77(1-3), 27-38 (2004).

[10] L. P. Institute, [Oregon State University].

[11] A. Caron, [Sunburn], (2004).

[12] K. K. Haruta Y, Yoshioka T, "Dietary Phospholipid from Bovine Milk Improves Epidermal Function in Hairless Mice," Bioscience, Biotechnology, Biochemistry, 72(2), 2151-7 (2008).

[13] E. M. Schmelz, "Dietary sphingomyelin and other sphingolipids in health and disease," Nutrition Foundation Nutrition Bulletin, 25, 135-139 (2000).

[14] M. Jun Choi, Maibachi H, "Role of ceramides in barrier function of healthy and diseased skin," American Journal of Clinical Dermatology, 6(4), 215-23 (2005).

[15] L. Coderch, Lopez, O., de la Maza, A., Parra, J, "Ceramides and skin function ” American Journal of Clinical Dermatology, 4(2), 107-129 (2003).

[16] E. Schmelz, Crall, K., Larocque, R., Dillehay, D. \& Merril, A, "Uptake and metabolism of sphingolipids in isolated intestinal loops of mice," The Journal of Nutrition, 124(5), 702-12 (1994).

[17] H. Vesper, Schmetz, E, "Sphingolipids in food and emerging importance of sphingolipids to nutrition," American Society of Nutritional Science, 129, 1239-50 (1999).

[18] D. Dillehay, Webb, S., Schmeltz, E. \& Merrill, A, "Dietary Sphingomyelin inhibits 1,2-dimethylhydrazineinduced colon cancer in CF1 mice," The Journal of Nutrition, 124(5), 615-20 (1993). 